\title{
QUANTILE MODELS WITH ENDOGENEITY
}

\author{
V. CHERNOZHUKOV AND C. HANSEN
}

\begin{abstract}
In this article, we review quantile models with endogeneity. We focus on models that achieve identification through the use of instrumental variables and discuss conditions under which partial and point identification are obtained. We discuss key conditions, which include monotonicity and full-rank-type conditions, in detail. In providing this review, we update the identification results of Chernozhukov and Hansen (2005). We illustrate the modeling assumptions through economically motivated examples. We also briefly review the literature on estimation and inference.

Key Words: identification, treatment effects, structural models, instrumental variables
\end{abstract}

\section{INTRODUCTION}

Quantile regression is a tool for estimating conditional quantile models that has been used in many empirical studies and has been studied extensively in theoretical econometrics; see Koenker and Bassett (1978) and Koenker (2005). One of quantile regression's most appealing features is its ability to estimate quantile-specific effects that describe the impact of covariates not only on the center but also on the tails of the conditional outcome distribution. While the central effects, such as the mean effect obtained through conditional mean regression, provide interesting summary statistics of the impact of a covariate, they fail to describe the full distributional impact unless the conditioning variables affect the central and the tail quantiles in the same way. In addition, researchers are interested in the impact of covariates on points other than the center of the conditional distribution in many cases. For example, in a study of the effectiveness of a job training program, the effect of training on the lower tail of the earnings distribution conditional on worker characteristics may be of more interest than the effect of training on the mean of the distribution.

In observational studies, the variables of interest (e.g. education or prices) are often endogenous. Just as with the conventional linear model, endogeneity of covariates renders

Date: First version: September 2011, this version March 29, 2013. We would like to thank the editor, Isaiah Andrews, Denis Chetverikov, and Ye Luo for excellent comments and much help. 
the conventional quantile regression inconsistent for estimating the causal (structural) effects of covariates on the quantiles of economic outcomes. One approach to addressing this problem is to generalize the instrumental variables framework to allow for estimation of quantile models. In this paper, we review developments in instrumental variables approaches to modeling and estimating quantile treatment (structural) effects (QTE) in the presence of endogeneity.

We focus our review on the modeling framework of Chernozhukov and Hansen (2005) which provides conditions for identification of the QTE without functional form assumptions. The principal identifying assumption of the model is the imposition of conditions which restrict how rank variables (structural errors) may vary across treatment states. These conditions allow the use of instrumental variables to overcome the endogeneity problem and recover the true QTE. This framework also ties naturally to simultaneous equations models, corresponding to a structural simultaneous equation model with nonadditive errors. Within this framework, estimation and inference procedures for linear quantile models have been developed by Chernozhukov and Hansen (2006), Chernozhukov and Hansen (2008), Chernozhukov, Hansen, and Jansson (2009), and Jun (2008); nonparametric estimation has been considered by Chernozhukov, Imbens, and Newey (2007), Horowitz and Lee (2007), and Gagliardini and Scaillet (2012); and inference with discrete outcomes has been explored by Chesher (2005). Moreover, the modeling framework provides a foundation for other estimation methods based on IV median-independence and more general quantile-independence conditions as in Abadie (1997), Chernozhukov and Hong (2003), Chen, Linton, and Keilegom (2003), Hong and Tamer (2003), Honore and $\mathrm{Hu}$ (2004), and Sakata (2007). It is also important to note that the modeling framework we review can be used to study nonparametric identification of structural economic models in cases where quantile effects are not necessarily the chief objects of interest. Berry and Haile (2010) provide an excellent example of this in the context of discrete choice models with endogeneity.

We also briefly review other modeling approaches for quantile effects with endogenous covariates. Abadie, Angrist, and Imbens (2002) consider a QTE model for the sub-population of "compliers" which applies to binary endogenous variables with binary instruments. Imbens and Newey (2009), Chesher (2003), Lee (2007), and Koenker and Ma (2006) use models with triangular structures and show how control functions can be constructed and used to estimate structural objects of interest. While these models share 
some features with the model of Chernozhukov and Hansen (2005), the three approaches are non-nested in general.

Quantile models with endogeneity have been used in many empirical studies in economics. See Abadie, Angrist, and Imbens (2002); Chernozhukov and Hansen (2004); Hausman and Sidak (2004); Forbes (2008); Eren (2009); Kostov (2009); Maynard and Qiu (2009); Wehby, Murran, Castilla, Lopez-Camelo, and Ohsfeldt (2009); Lamarche (2011); Autor, Houseman, and Kerr (2012); and Somainiy (2012) among others. We do not provide a review of empirical applications but note these papers provide further discussion of how the instrumental variables quantile model relates to their specific framework and illustrate some of the rich effects that one can estimate using quantile methods.

\section{An IV Quantile Model}

In this section, we present an instrumental variable model for quantile treatment effects (QTE), its main econometric implication, and the principal identification result.

2.1. Framework. Our model is developed within the conventional potential (latent) outcome framework, e.g. Heckman and Robb (1986). Potential real-valued outcomes which vary among individuals or observational units are indexed against potential treatment states $d \in \mathcal{D}$ and denoted $Y_{d}$. The potential outcomes $\left\{Y_{d}\right\}$ are latent because, given the selected treatment $D$, the observed outcome for each individual or observational unit is only one component

$$
Y:=Y_{D}
$$

of the potential outcomes vector $\left\{Y_{d}\right\}$. Throughout the paper, capital letters denote random variables, and lower case letters denote the potential values they may take. We do not explicitly state various technical measurability assumptions as these can be deduced from the context 1

The objective of causal or structural analysis is to learn about features of the distributions of potential outcomes $Y_{d}$. Of primary interest to us are the $\tau$-th quantiles of potential outcomes under various treatments $d$, conditional on observed characteristics $X=x$, denoted as

$$
q(d, x, \tau) .
$$

\footnotetext{
${ }^{1}$ For simplicity, we could assume that $d$ takes on a countable set of values $\mathcal{D}$ or make separability assumptions which imply that the stochastic process $\left\{Y_{d}, d \in \mathcal{D}\right\}$ is defined from its definition over a countable subset $\mathcal{D}_{0} \subset \mathcal{D}$. See van der Vaart and Wellner (1996).
} 
We will refer to the function $q(d, x, \tau)$ as the quantile treatment response (QTR) function. We are also interested in the quantile treatment effects (QTE), defined as

$$
q\left(d_{1}, x, \tau\right)-q\left(d_{0}, x, \tau\right)
$$

that summarize the differences in the impact of treatments on the quantiles of potential outcomes (Lehmann (1974), Doksum (1974)).

Typically, the realized treatment $D$ is selected in relation to potential outcomes, inducing endogeneity. This endogeneity makes the conventional quantile regression of observed $Y$ on observed $D$, which relies upon the restriction

$$
P[Y \leqslant \theta(D, X, \tau) \mid X, D]=\tau \text { a.s. }
$$

inappropriate for measuring $q(d, x, \tau)$ and the QTE. Indeed the function $\theta(d, x, \tau)$ solving these equations will not be equal to $q(d, x, \tau)$ under endogeneity. The model presented next states conditions under which we can identify and estimate the quantiles of latent outcomes through the use of instruments $Z$ that affect $D$ but are independent of potential outcomes and the nonlinear quantile-type conditional moment restrictions

$$
P[Y \leqslant q(D, X, \tau) \mid X, Z]=\tau \text { a.s. }
$$

2.2. The Instrumental Quantile Treatment Effects (IVQT) Model. Having conditioned on the observed characteristics $X=x$, each latent outcome $Y_{d}$ can be related to its quantile function $q(d, x, \tau)$ as 2

$$
Y_{d}=q\left(d, x, U_{d}\right), \text { where } U_{d} \sim U(0,1)
$$

is the structural error term. We note that representation (2.1) is essential to what follows.

The structural error $U_{d}$ is responsible for heterogeneity of potential outcomes among individuals with the same observed characteristics $x$. This error term determines the relative ranking of observationally equivalent individuals in the distribution of potential outcomes given the individuals' observed characteristics, and thus we refer to $U_{d}$ as the rank variable. Since $U_{d}$ drives differences in observationally equivalent individuals, one may think of $U_{d}$ as representing some unobserved characteristic, e.g. ability or proneness 3 This interpretation makes quantile analysis an interesting tool for describing and

\footnotetext{
${ }^{2}$ This follows by Fisher-Skorohod representation of random variables which states that given a collection of variables $\left\{\zeta_{d}\right\}$, each variable $\zeta_{d}$ can be represented as $\zeta_{d}=q\left(d, U_{d}\right)$, for some $U_{d} \sim U(0,1)$, cf. Durrett (1996), where $q(d, \tau)$ denotes the $\tau$-quantile of variable $\zeta_{d}$.

${ }^{3}$ Doksum (1974) uses the term proneness as in "prone to learn fast" or "prone to grow taller".
} 
learning the structure of heterogeneous treatment effects and accounting for unobserved heterogeneity; see Doksum (1974), Heckman and Smith (1997), and Koenker (2005).

For example, consider a returns-to-training model, where $Y_{d}$ 's are potential earnings under different training levels $d$, and $q(d, x, \tau)$ is the conditional earnings function which describes how an individual having training $d$, characteristics $x$, and the latent "ability" $\tau$ is rewarded by the labor market. The earnings function may be different for different levels of $\tau$, implying heterogeneous effects of training on earnings of people that have different levels of "ability". For example, it may be that the largest returns to training accrue to those in the upper tail of the conditional distribution, that is, to the "high-ability" workers 4

Formally, the IVQT model consists of five conditions (some are representations) that hold jointly.

Main Conditions of the Model: Consider a common probability space $(\Omega, F, P)$ and the set of potential outcome variables $\left(Y_{d}, d \in \mathcal{D}\right)$, the covariate variables $X$, and the instrumental variables $Z$. The following conditions hold jointly with probability one:

A1 Potential Outcomes. Conditional on $X$ and for each $d, Y_{d}=q\left(d, X, U_{d}\right)$, where $\tau \mapsto q(d, X, \tau)$ is non-decreasing on $[0,1]$ and left-continuous and $U_{d} \sim U(0,1)$.

A2 Independence. Conditional on $X$ and for each $d, U_{d}$ is independent of instrumental variables $Z$.

A3 Selection. $D:=\delta(Z, X, V)$ for some unknown function $\delta$ and random vector $V$.

A4 Rank Similarity. Conditional on $(X, Z, V),\left\{U_{d}\right\}$ are identically distributed.

A5 OBserved random vector consists of $Y:=Y_{D}, D, X$ and $Z$.

The following is the main econometric implication of the model.

\footnotetext{
${ }^{4}$ It is important to note that the quantile index, $\tau$, in $q(d, x, \tau)$ refers to the quantile of potential outcome $Y_{d}$ given that exogenous variables are set at $X=x$ and not to the unconditional quantile of $Y_{d}$. For example, suppose that one of the control variables in the earnings example is years of schooling. An individual at the $30^{\text {th }}$ percentile of the distribution of $Y_{d}$ given say 20 years of schooling is not necessarily low income as even a relatively low earner with that level of education may still earn above the median earnings in the overall population.
} 
Theorem 1 (Main Statistical Implication). Suppose conditions A1-A5 hold. (i) Then we have for $U:=U_{D}$, with probability one,

$$
Y=q(D, X, U), \quad U \sim U(0,1) \mid X, Z .
$$

(ii) If (2.2) holds and $\tau \mapsto q(d, \tau)$ is strictly increasing for each $d$, then for each $\tau \in(0,1)$, a.s

$$
P[Y \leqslant q(D, X, \tau) \mid X, Z]=\tau .
$$

(iii) If (2.2) holds, then for any closed subset I of $[0,1]$, a.s.

$$
P(U \in I) \leqslant P[Y \in q(D, X, I) \mid X, Z],
$$

where $q(d, x, I)$ is the image of I under the mapping $\tau \mapsto q(d, x, \tau)$.

The first result states that the main consequence of A1-A5 is a simultaneous equation model (2.2) with non-separable error $U$ that is independent of $Z, X$, and normalized so that $U \sim U(0,1)$. The second result considers econometric implications when $\tau \mapsto$ $q(D, X, \tau)$ is strictly increasing, which requires that $Y$ is non-atomic conditional on $X$ and $Z$. In this case, we obtain the conditional moment restriction (2.3). This implication follows from the first result and the fact that

$$
\{Y \leqslant q(D, X, \tau)\} \text { is equivalent to }\{U \leqslant \tau\},
$$

when $q(D, X, \tau)$ is strictly increasing in $\tau$. The final result deals with the case where $Y$ may have atoms conditional on $X$ and $Z$, e.g. when $Y$ is a count or discrete response variable. The first two results were obtained in Chernozhukov and Hansen (2005), and the third result is in the spirit of results given in Chesher, Rosen, and Smolinski (2011); Chesher (2005); and Chesher and Smolinski (2010). The latter results are related to random set/optimal transport methods for identification analysis; see Beresteanu, Molchanov, and Molinari (2011); Ekeland, Galichon, and Henry (2010); Galichon and Henry (2009); and Galichon and Henry (2011).

The model and the results of Theorem 1 are useful for two reasons. First, Theorem 1 serves as a means of identifying the QTE in a reasonably general heterogeneous effects model. Second, by demonstrating that the IVQT model leads to the conditional moment restrictions (2.3) and (2.4), Theorem 1 provides an economic and causal foundation for estimation based on these restrictions. 
2.3. The Identification Regions. The conditions presented above yield the following identification region for the structural quantile function $(d, x, \tau) \mapsto q(d, x, \tau)$. The identification region for the case of strictly increasing $\tau \mapsto q(d, x, \tau)$ can be stated as the set $\mathcal{Q}$ of functions $(d, x, \tau) \mapsto m(d, x, \tau)$ that satisfy the following relations, for all $\tau \in(0,1]$

$$
P[Y<m(D, X, \tau) \mid X, Z]=\tau \text { a.s. }
$$

This representation of the identification region $\mathcal{Q}$ is implicit. Nevertheless, statistical inference about $q \in \mathcal{Q}$ can be based on (2.5) and can be carried out in practice using weak-identification robust inference as described in Chernozhukov and Hansen (2008), Marmer and Sakata (2012), Jun (2008), Santos (2012), or Chernozhukov, Hansen, and Jansson (2009). Under conditions that yield point identification, these regions collapse to a singleton, and the aforementioned weak-identification-robust inference procedures retain their validity.

The identification region for the case of weakly increasing $\tau \mapsto q(d, x, \tau)$ can be stated as the set $\mathcal{Q}$ of functions $(d, x, \tau) \mapsto m(d, x, u)$ that satisfy the following relations: For any closed subset $I$ of $(0,1]$,

$$
P(U \in I) \leqslant P[Y \in m(D, X, I) \mid X, Z] \text { a.s., }
$$

where $m(D, X, I)$ is the image of $I$ under the mapping $\tau \mapsto m(D, X, \tau)$. The inference problem here falls in the class of conditional moment inequalities and approaches such as those described in Andrews and Shi (2013) or Chernozhukov, Lee, and Rosen (2013), for example, can be used. The sets $I$ to be checked could be reduced by determining approximate core-determining subsets; see Chesher, Rosen, and Smolinski (2011), Galichon and Henry (2009), Galichon and Henry (2011) for further discussion.

2.4. Discussion of the Model. Condition A1 imposes monotonicity on the structural function of interest which makes its relation to the QTR apparent. Condition A2 states that potential outcomes are independent of $Z$, given $X$, which is a conventional independence restriction. Condition $A 3$ is a convenient representation of a treatment selection mechanism, stated for the purposes of discussion. In A3, the unobserved random vector $V$ is responsible for the difference in treatment choices $D$ across observationally identical individuals. Dependendence between $V$ and $\left\{U_{d}\right\}$ is the source of endogeneity that makes the conventional exogeneity assumption $U \sim U(0,1) \mid X, D$ break down. This failure leads to inconsistency of exogenous quantile methods for estimating the structural quantile 
function. Within the model outlined above, this breakdown is resolved through the use of instrumental variables.

The independence imposed in A2 and A3 is weaker than the commonly made assumption that both the disturbances $\left\{U_{d}\right\}$ in the outcome equation and the disturbances $V$ in the selection equation are jointly independent of the instrument $Z$; e.g. Heckman and Robb (1986) and Imbens and Angrist (1994). The latter assumption may be violated when the instrument is measured with error as discussed in Hausman (1977) or the instrument is not assigned exogenously relative to the selection equation as in Example 2 in Imbens and Angrist (1994).

Condition A4 restricts the variation in ranks across potential outcomes and is key for identifying the QTR and associated QTE. Its simplest, though strongest, form is rank invariance, when ranks $U_{d}$ do not vary with potential treatment states $d: 5$

$$
U_{d}=U \text { for each } d \in \mathcal{D} \text {. }
$$

For example, under rank invariance, people who are strong (highly ranked) earners without a training program $(d=0)$ remain strong earners having done the training $(d=1)$. Indeed, the earnings of a person with characteristics $x$ and rank $U=\tau$ in the training state " 0 " is $Y_{0}=q(0, x, \tau)$ and in the state " 1 " is $Y_{1}=q(1, x, \tau) 6$ Thus, rank invariance implies that a common unobserved factor $U$, such as innate ability, determines the ranking of a given person across treatment states.

Rank invariance implies that the potential outcomes $\left\{Y_{d}\right\}$ are jointly degenerate which may be implausible on logical grounds, as pointed out by Heckman and Smith (1997). Also, the rank variables $U_{d}$ may be determined by many unobserved factors. Thus, it is desirable to allow the rank $U_{d}$ to change across $d$, reflecting some unobserved, asystematic variation. Rank similarity A4 achieves this property while managing to preserve the useful moment restriction (2.3).

Rank similarity A4 relaxes exact rank invariance by allowing asystematic deviations, "slippages" in the terminology of Heckman and Smith (1997), in one's rank away from some common level $U$. Conditional on $U$, which may enter disturbance $V$ in the selection

\footnotetext{
${ }^{5}$ Notice that under rank invariance, condition A3 is a pure representation, not a restriction, since nothing restricts the unobserved information component $V$.

${ }^{6}$ Rank invariance is used in many interesting models without endogeneity. See e.g. Doksum (1974), Heckman and Smith (1997), and Koenker and Geling (2001).
} 
equation, we have the following condition on the slippages 7

$$
U_{d}-U \text { are identically distributed across } d \in \mathcal{D} \text {. }
$$

In this formulation, we implicitly assume that one selects the treatment without knowing the exact potential outcomes; i.e. one may know $U$ and even the distribution of slippages, but does not know the exact slippages $U_{d}-U$. This assumption is consistent with many empirical situations where the exact latent outcomes are not known before receipt of treatment. We also note that conditioning on appropriate covariates $X$ may be important to achieve rank similarity.

In summary, rank similarity is an important restriction of the IVQT model that allows us to address endogeneity. This restriction is absent in conventional endogenous heterogeneous treatment effect models. However, similarity enables a more general selection mechanism, A3, and weaker independence conditions on instruments than often are assumed in nonseparable IV models. The main force of rank similarity and the other stated assumptions is the implied moment restriction (2.3) of Theorem 1, which is useful for identification and estimation of the quantile treatment effects.

2.5. Examples. We present some examples that highlight the nature of the model, its strengths, and its limitations.

Example 1 (Demand with Non-Separable Error). The following is a generalization of the classic supply-demand example. Consider the model

$$
\begin{aligned}
& Y_{p}=q(p, U), \\
& \tilde{Y}_{p}=\rho(p, z, \mathcal{U}), \\
& P \in\{p: \rho(p, Z, U)=q(p, \mathcal{U})\},
\end{aligned}
$$

where functions $q$ and $\rho$ are increasing in the last argument. The function $p \mapsto Y_{p}$ is the random demand function, and $p \mapsto \tilde{Y}_{p}$ is the random supply function. Additionally, functions $q$ and $\rho$ may depend on covariates $X$, but this dependence is suppressed.

Random variable $U$ is the level of demand and describes the demand curve at different states of the world. Demand is maximal when $U=1$ and minimal when $U=0$, holding $p$ fixed. Note that we imposed rank invariance (2.7), as is typical in classic supply-demand models, by making $U$ invariant to $p$.

\footnotetext{
${ }^{7}$ Conditioning is required to be on all components of $V$ in the selection equation A3.
} 
Model (2.9) incorporates traditional additive error models for demand which have $Y_{p}=$ $q(p)+\epsilon$ where $\epsilon=Q_{\epsilon}(U)$. The model is much more general in that the price can affect the entire distribution of the demand curve, while in traditional models it only affects the location of the distribution of the demand curve.

The $\tau$-quantile of the demand curve $p \mapsto Y_{p}$ is given by $p \mapsto q(p, \tau)$. Thus, the curve $p \mapsto Y_{p}$ lies below the curve $p \mapsto q(p, \tau)$ with probability $\tau$. Therefore, the various quantiles of the potential outcomes play an important role in describing the distribution and heterogeneity of the stochastic demand curve. The quantile treatment effect may be characterized by $\partial q(p, \tau) / \partial p$ or by an elasticity $\partial \ln q(p, \tau) / \partial \ln p$. For example, consider the Cobb-Douglas model $q(p, \tau)=\exp (\beta(\tau)+\alpha(\tau) \ln p)$ which corresponds to a CobbDouglas model for demand with non-separable error $Y_{p}=\exp (\beta(U)+\alpha(U) \ln p)$. The $\log$ transformation gives $\ln Y_{p}=\beta(U)+\alpha(U) \ln p$, and the quantile treatment effect for the $\log$-demand equation is given by the elasticity of the original $\tau$-demand curve $\alpha(\tau)=$ $\frac{\partial Q_{\ln Y_{p}(\tau)}}{\partial \ln p}=\frac{\partial \ln q(p, \tau)}{\partial \ln p}$.

The elasticity $\alpha(U)$ is random and depends on the state of the demand $U$ and may vary considerably with $U$. For example, this variation could arise when the number of buyers varies and aggregation induces a non-constant elasticity across the demand levels. Chernozhukov and Hansen (2008) estimate a simple demand model based on data from a New York fish market that was first collected and used by Graddy (1995). They find point estimates of the demand elasticity, $\alpha(\tau)$, that vary quite substantially from -2 for low quantiles to -0.5 for high quantiles of the demand curve.

The third condition in (3.3), $P \in\{p: \rho(p, Z, U)=q(p, \mathcal{U})\}$, is the equilibrium condition that generates endogeneity; the selection of the clearing price $P$ by the market depends on the potential demand and supply outcomes. As a result we have a representation that is consistent with $\mathrm{A} 3, P=\delta(Z, V)$, where $V$ consists of $U$ and $\mathcal{U}$ and may include "sunspot" variables if the equilibrium price is not unique. Thus what we observe can be written as

$$
Y:=q(P, U), \quad P:=\delta(Z, V), \quad U \text { is independent of } Z .
$$

Identification of the $\tau$-quantile of the demand function, $p \mapsto q(p, \tau)$ is obtained through the use of instrumental variables $Z$, like weather conditions or factor prices, that shift the supply curve and do not affect the level of the demand curve, $U$, so that independence assumption A2 is met. Furthermore, the IVQT model allows arbitrary correlation between $Z$ and $V$. This property is important as it allows, for example, $Z$ to be measured with 
error or to be exogenous relative to the demand equation but endogeneous relative to the supply equation.

Example 2 (Savings). Chernozhukov and Hansen (2004) use the framework of the IVQT model to examine the effects of participating in a 401(k) plan on an individual's accumulated wealth. Since wealth is continuous, wealth, $Y_{d}$, in the participation state $d \in\{0,1\}$ can be represented as

$$
Y_{d}=q\left(d, X, U_{d}\right), \quad U_{d} \sim U(0,1)
$$

where $\tau \mapsto q(d, X, \tau)$ is the conditional quantile function of $Y_{d}$ and $U_{d}$ is an unobserved random variable. $U_{d}$ is an unobservable that drives differences in accumulated wealth conditional on $X$ under participation state $d$. Thus, one might think of $U_{d}$ as the preference for saving and interpret the quantile index $\tau$ as indexing rank in the preference for saving distribution. One could also model the individual as selecting the 401(k) participation state to maximize expected utility:

$$
D=\arg \max _{d \in \mathcal{D}} E\left[W\left\{Y_{d}, d\right\} \mid X, Z, V\right]=\arg \max _{d \in \mathcal{D}} E\left[W\left\{q\left(d, x, U_{d}\right), d\right\} \mid X, Z, V\right],
$$

where $W\left\{Y_{d}, d\right\}$ is the random indirect utility derived under participation state $d .8$ As a result, the participation decision is represented by

$$
D=\delta(Z, X, V)
$$

where $Z$ and $X$ are observed, $V$ is an unobserved information component that may be related to ranks $U_{d}$ and includes other unobserved variables that affect the participation state, and function $\delta$ is unknown. This model fits into the IVQT model with the independence condition A2 requiring that $U_{d}$ is independent of $Z$, conditional on $X$.

The simplest form of rank similarity is rank invariance (2.7), under which the preference for saving vector $U_{d}$ may be collapsed to a single random variable $U=U_{0}=U_{1}$. In this case, a single preference for saving is responsible for an individual's ranking across all treatment states. The rank similarity condition A4 is a more general form of rank invariance. It relaxes the exact invariance of ranks $U_{d}$ across $d$ by allowing noisy, unsystematic variations of $U_{d}$ across $d$, conditional on $(V, X, Z)$. This relaxation allows for variation in rank across the treatment states, requiring only an "expectational rank invariance." Similarity implies that given the information in $(V, X, Z)$ employed to make the selection of treatment $D$, the expectation of any function of rank $U_{d}$ does not vary across the treatment states. That is, ex-ante, conditional on $(V, X, Z)$, the ranks may be considered to

\footnotetext{
${ }^{8}$ It may depend on both observables in $X$ as well as realized and unrealized unobservables. Only dependence on $Y_{d}$ and $d$ is highlighted.
} 
be the same across potential treatments, but the realized, ex-post, rank may be different across treatment states.

From an econometric perspective, the similarity assumption is nothing but a restriction on the unobserved heterogeneity component which precludes systematic variation of $U_{d}$ across the treatment states. To be more concrete, consider the following simple example where

$$
U_{d}=F_{V+\eta_{d}}\left(V+\eta_{d}\right)
$$

where $F_{V+\eta_{d}}(\cdot)$ is the distribution function of $V+\eta_{d}$ and $\left\{\eta_{d}\right\}$ are mutually iid conditional on $V, X$, and $Z$. The variable $V$ represents an individual's "mean" saving preference, while $\eta_{d}$ is a noisy adjustment 9 This more general assumption leaves the individual optimization problem (2.11) unaffected, while allowing variation in an individual's rank across different potential outcomes.

While we feel that similarity may be a reasonable assumption in many contexts, imposing similarity is not innocuous. In the context of 401(k) participation, matching practices of employers could jeopardize the validity of the similarity assumption. To be more concrete, let $U_{d}=F_{V+\eta_{d}}\left(V+\eta_{d}\right)$ as before but let $\eta_{d}=d M$ for random variable $M$ that depends on the match rate and is independent of $V, X$, and $Z$. Then conditional on $V=v, X$, and $Z, U_{0}=F_{V}(v)$ is degenerate but $U_{1}=F_{V+M}(v+M)$ is not. Therefore, $U_{1}$ is not equal to $U_{0}$ in distribution. Similarity may still hold in the presence of the employer match if the rank, $U_{d}$, in the asset distribution is insensitive to the match rate. The rank may be insensitive if, for example, individuals follow simple rules of thumb such as target saving when they make their savings decisions. Also, if the variation of match rates is small relative to the variation of individual heterogeneity or if the covariates capture most of the variation in match rates, then similarity may be satisfied approximately.

Example 3 (Discrete Choice Model with Market-Level Data). Berry and Haile (2010) show that a general model for market-level data realized from a discrete-choice problem can fit within the IVQT model. To keep notation and exposition simple, we consider a much-simplified version of the model from Berry and Haile (2010) in which consumer $i$ 's indirect utility from choosing product $j$ is

$$
U_{i j t}=u\left(X_{j t}, P_{j t}, \xi_{j t}, V_{i j t}\right)=u\left(\delta_{j}\left(X_{j t}, \xi_{j t}\right), P_{j t}, V_{i j t}\right),
$$

where $t$ indexes markets, $X_{j t}$ are observed exogenous product-market characteristics, $P_{j t}$ is the observed price of product $j$ in market $t$ which is treated as endogenous, $\xi_{j t}$ are

\footnotetext{
${ }^{9}$ Clearly similarity holds in this case, $U_{d} \stackrel{d}{=} U_{d^{\prime}}$ given $V, X$, and $Z$.
} 
product-market specific unobservables, and $V_{i j t}$ are individual-product-market specific unobservables that have density $f(\cdot)$. Thus, the model imposes that unobserved productmarket specific effects and observed variables $X_{j t}$ may only affect utility through the index $\delta_{j t}=\delta_{j}\left(X_{j t}, \xi_{j t}\right)$, where $\delta_{j}(\cdot, \cdot)$ may differ arbitrarily across products but is the same across all markets. That unobserved product characteristics affect utility only through a scalar index is a substantive restriction but is common in the literature on discrete choice models where, for example, one can interpret the index as an aggregate representing product quality.

An individual will then choose the product that maximizes individual utility. Letting $Y_{i t}$ denote the observed choice of individual $i$, we have that

$$
Y_{i t}=\arg \max _{j \leq J} U_{i j t}
$$

where we assume the same $J$ products are available in each market for simplicity 10 The market share of each product will then be given as

$$
\begin{aligned}
S_{j t} & =\int 1\left\{u\left(\delta_{j t}, P_{j t}, v\right)=\max _{k \leqslant J} u\left(\delta_{k t}, P_{k t}, v\right)\right\} f(v) d v \\
& :=s_{j}\left(\left\{\delta_{j t}, P_{j t}\right\}_{j=1}^{J}\right)=s_{j}\left(\delta_{t}, P_{t}\right),
\end{aligned}
$$

where $\delta_{t}=\left(\delta_{1 t}, \ldots, \delta_{J t}\right)^{\prime}$ and $P_{t}=\left(P_{1 t}, \ldots, P_{J t}\right)^{\prime}$.

To fit this model into the instrumental variables quantile regression model, Berry and Haile (2010) make several assumptions to produce a structural relationship which is monotonic in a scalar unobservable. First, they assume that the utility function $u\left(\delta_{j t}, P_{j t}, V_{i j t}\right)$ is strictly increasing in $\delta_{j t}$. This assumption is standard in the discrete choice literature and coincides with the interpretation of $\delta_{j t}$ as product quality where higher quality products are associated with higher utility all else equal. Monotonicity of the utility function is not sufficient due to the fact that all that is observed is the market share which depends on the utility of each potential choice. Thus, Berry and Haile (2010) make an additional assumption that they term "connected substitutes." Intuitively, this condition implies that an increase in the quality of every good within some strict subset of the available choices will be associated with the total market share of all goods not in the subset decreasing as long as the quality of no good outside of the subset increases. Berry and Haile

\footnotetext{
${ }^{10}$ Obviously, identification of the model requires normalizations. For example, the utility from one of the options is generally normalized to zero. As this model is not the focus of this review, we do not discuss these normalizations which are discussed in detail in a more general context in Berry and Haile (2010).
} 
(2010) show that the connected substitutes condition is satisfied in usual random utility discrete choice models and that it can hold fairly generally. Using these assumptions, Berry and Haile (2010) use a result from Gandhi (2008) which shows that the system of equations

$$
S_{j t}=s_{j}\left(\delta_{t}, P_{t}\right)
$$

has a unique solution for the vector $\delta_{t}$ as long as all goods present in equilibrium have positive market shares. Thus, we may write

$$
\delta_{j t}=g_{j}\left(S_{t}, P_{t}\right)
$$

for some function $g_{j}$ where $S_{t}=\left(S_{1 t}, \ldots, S_{J t}\right)^{\prime}$.

From (2.12), we have that $\delta_{j}\left(X_{j t}, \xi_{j t}\right)=g_{j}\left(S_{t}, P_{t}\right)$. To complete the argument, Berry and Haile (2010) assume that the function $\delta_{j}\left(X_{j t}, \xi_{j t}\right)$ is strictly increasing in its second argument, $\xi_{j t}$, which represents unobserved product attributes. This condition rules out the case where $\xi_{j t}$ can represent attributes that would increase utility for some individuals but decrease utility for others and again corresponds to the notion that $\xi_{j t}$ represents unobserved product quality in which an increase unambiguously makes the product more desirable. With the assumed monotonicity in the function $\delta_{j}$, one obtains

$$
\xi_{j t}=\delta_{j}^{-1}\left(g_{j}\left(S_{t}, P_{t}\right) ; X_{j t}\right)=h_{j}\left(S_{t}, P_{t}, X_{j t}\right) .
$$

It is also clear that $h_{j}\left(X_{t}, P_{t}, S_{t}\right)$ is strictly increasing in $S_{j t}$, which is proven in Lemma 5 of Berry and Haile (2010), from which it follows that

$$
S_{j t}=q_{j}\left(S_{-j t}, P_{t}, X_{j t}, \xi_{j t}\right)
$$

where $S_{-j t}$ denotes the set of market shares for each product in market $t$ excluding product $j$ and $q_{j}$ is an unknown function that is strictly increasing in $\xi_{j t}$. Then, $q_{j}$ can be

taken as the structural function in the instrumental variables quantile model after the normalization that $\xi_{j t}$ follows a $U(0,1)$, assuming that $\xi_{j t}$ has an atomless distribution. The model is then completed by assuming the existence of instruments, $Z_{t}$, that are independent of $\xi_{j t}$ conditional on $X_{j t}$ and are related to the endogenous variables through $\left(S_{-j t}^{\prime}, P_{t}^{\prime}\right)^{\prime}=\Delta\left(Z_{t}, X_{j t}, V_{t}\right)$ for some function $\Delta$ and unobservables $V_{t}$. Finally, note that the model assumes rank invariance in its construction.

\section{The Identifying Power of IV Quantile Restrictions}

The purpose of this section is to examine the identifying power of conditional moment restrictions (2.3) . Specifically, we give various conditions for point identification in this 
section, summarizing and updating some of the results known in the literature. We remark here that point identification is not required in applications in principle as there exist inference methods that apply without point identification. However, it is useful to know and understand conditions under which moment conditions are informative enough that the identification region shrinks to a single point; in such cases the inference methods will also produce very informative confidence sets. We present point-identifying conditions first for the binary case, $D \in\{0,1\}$ and $Z \in\{0,1\}$, and then consider the case of $D$ taking a finite number of values, and finally consider the continuous case.

3.1. Conditions for point identification in the binary case. Here we consider the cases where $D \in\{0,1\}$ and $Z \in\{0,1\}$. The following analysis is all conditional on $X=x$ and for a given quantile $\tau \in(0,1)$, but we suppress this dependence for ease of notation. Under the conditions of Theorem 1, we know that there is at least one function $q(d):=q(d, x, \tau)$ that solves $P[Y \leqslant q(D) \mid Z]=\tau$ a.s. The function $q(\cdot)$ can be equivalently represented by a vector of its values $q=(q(0), q(1))^{\prime}$. Therefore, for vectors of the form $y=\left(y_{0}, y_{1}\right)^{\prime}$, we have a vector of moment equations

$$
\Pi(y):=\left(P\left[Y \leqslant y_{D} \mid Z=0\right]-\tau, P\left[Y \leqslant y_{D} \mid Z=1\right]-\tau\right)^{\prime}
$$

where $y_{D}:=(1-D) \cdot y_{0}+D \cdot y_{1}$. We say that $q$ is identified in some parameter space, $\mathcal{L}$, if $y=q$ is the only solution to $\Pi(y)=0$ among all $y \in \mathcal{L}$.

We require that the Jacobian $\partial \Pi(y)$ of $\Pi(y)$ with respect to $y=\left(y_{0}, y_{1}\right)^{\prime}$ exists and that it takes the form

$$
\begin{aligned}
\partial \Pi(y):= & {\left[\begin{array}{ll}
f_{Y}\left(y_{0} \mid D=0, Z=0\right) P[D=0 \mid Z=0] & f_{Y}\left(y_{1} \mid D=1, Z=0\right) P[D=1 \mid Z=0] \\
f_{Y}\left(y_{0} \mid D=0, Z=1\right) P[D=0 \mid Z=1] & f_{Y}\left(y_{1} \mid D=1, Z=1\right) P[D=1 \mid Z=1]
\end{array}\right] } \\
= & :\left[\begin{array}{ll}
f_{Y, D}\left(y_{0}, 0 \mid Z=0\right) & f_{Y, D}\left(y_{1}, 1 \mid Z=0\right) \\
f_{Y, D}\left(y_{0}, 0 \mid Z=1\right) & f_{Y, D}\left(y_{1}, 1 \mid Z=1\right)
\end{array}\right] .
\end{aligned}
$$

For local identification, we take $\mathcal{L}$ as an open neighborhood of $q=(q(0), q(1))^{\prime}$. For global identification, we shall use some definitions from Mas-Collell to define $\mathcal{L}$. In what follows, for every proper (non-null) subspace $L \subset \mathbb{R}^{l}$, let $\operatorname{proj}_{L}: \mathbb{R}^{l} \mapsto L$ denote the perpendicular projection map. A convex, compact polytope is a bounded convex set formed by an intersection of a finite number of closed half-spaces. Such a polytope is of full dimension in $\mathbb{R}^{l}$ if it has a non-empty interior in $\mathbb{R}^{l}$. A face of a polytope $\mathcal{L}$ is the intersection of any supporting hyperplane of $\mathcal{L}$ with $\mathcal{L}$, so that faces of a polytope necessarily include the polytope itself. For instance, a rectangle in $\mathbb{R}^{2}$ has one 2-dimensional face 
given by itself, four 1-dimensional faces given by its edges, and four 0-dimensional faces gives by its vertices. A subspace spanned by a non-empty face of $\mathcal{L}$ is the translation to the origin of the minimal affine space containing that face.

Theorem 2 (Identification by Full Rank Conditions). Suppose that $\Pi(q)=0$, the support of $D$ is $\{0,1\}$ and the support of $Z$ is $\{0,1\}$. Assume that the conditional density $f_{Y}(y \mid D=$ $d, Z=z$ ) exists for each $y \in \mathbb{R}$ and $(d, z) \in\{0,1\} \times\{0,1\}$. (i) (Local) Suppose the Jacobian $\partial \Pi$ given by (3.14) is continuous and has full rank at $y=q$, then the $\tau$-quantiles of potential outcomes, $q=(q(0), q(1))^{\prime}$, are identified in the region $\mathcal{L}$ given by a sufficiently small open neighborhood of $q$ in $\mathbb{R}^{2}$. (ii) (Global) Assume that region $\mathcal{L}$ contains $q$ and can be covered by a finite number of compact convex 2-dimensional polytopes $\left\{\mathcal{L}_{j}\right\}$, each containing q. Assume that for each $j$, $\partial \Pi$ is a $C^{1}$ Jacobian of $\Pi: \mathcal{L}_{j} \rightarrow \mathbb{R}^{2}$, and that, possibly after rearranging the rows of $\partial \Pi$, for each $y \in \mathcal{L}_{j}$ and each subspace $L \subset \mathbb{R}^{2}$ spanned by a face of $\mathcal{L}_{j}$ that includes $y$, the linear map

$$
\operatorname{proj}_{L} \circ \partial \Pi(y): L \mapsto L
$$

has a positive determinant. Then $q$ is identified in $\mathcal{L}$.

The first result is a simple local identification condition of the type considered in Rothenberg (1971) which we provide to fix ideas. The second result is a global identification condition which extends the result in Chernozhukov and Hansen (2005) by allowing non-rectangular sets $\mathcal{L}$. This result is based on the global univalence theorems of Mas-Colell (1979). As explained below, the positive determinant condition requires the impact of instrument $Z$ on the joint distribution of $(Y, D)$ to be sufficiently rich. In particular, the instrument $Z$ should not be independent of the endogenous variable $D$. We note that existence of the conditional density $f_{Y}(y \mid D=d, Z=z)$ is only required for $(d, z)$ in the support of $(D, Z)$. Outside the support we can define the conditional density as 0 , so the existence condition is not very restrictive. Moreover, the condition is formulated so that $\mathcal{L}$ can take on relatively rich shapes that can carry useful economic restrictions. For instance, in the training context, a useful restriction on the parameters is that training weakly increases the potential earning quantiles. This restriction can be implemented by taking some natural parameter space and intersecting it with the half-space $H=\left\{\left(y_{0}, y_{1}\right) \in \mathbb{R}^{2}: y_{1} \geqslant y_{0}\right\}$. Specifically, a cube $C=\left\{y \in \mathbb{R}^{l}:\|y\|_{\infty} \leqslant K\right\}$ intersected with the halfspace $H$ is an example of a region $\mathcal{L}$ permitted by the global identification result (ii). 
Comment 3.1 (Simple Sufficient Conditions). To illustrate the conditions of the theorem, let us consider the parameter space $\mathcal{L}$ as either $\mathcal{L}=q+C$, i.e. a cube centered at $q$, or $\mathcal{L}=(q+C) \cap H$, i.e. intersection of a cube centered at $q$ with the halfspace $H$. Consider the trivial covering of $\mathcal{L}$ by itself, i.e. $\mathcal{L}_{j}=\mathcal{L}$. Then the positive determinant condition of the theorem is implied by the following simple conditions:

$$
\frac{f_{Y, D}\left(y_{1}, 1 \mid Z=1\right)}{f_{Y, D}\left(y_{0}, 0 \mid Z=1\right)}>\frac{f_{Y, D}\left(y_{1}, 1 \mid Z=0\right)}{f_{Y, D}\left(y_{0}, 0 \mid Z=0\right)} \text { for all } y=\left(y_{0}, y_{1}\right) \in \mathcal{L},
$$

and

$$
f_{Y, D}\left(y_{1}, 1 \mid Z=1\right)>0, \quad f_{Y, D}\left(y_{0}, 0 \mid Z=0\right)>0, \quad \text { for all } y=\left(y_{0}, y_{1}\right) \in \mathcal{L}
$$

Alternatively, since we can rearrange the rows of $\partial \Pi$, which corresponds to reordering elements of vector $\Pi$, the positive determinant condition of the theorem is implied by the following simple conditions:

$$
\frac{f_{Y, D}\left(y_{1}, 1 \mid Z=1\right)}{f_{Y, D}\left(y_{0}, 0 \mid Z=1\right)}<\frac{f_{Y, D}\left(y_{1}, 1 \mid Z=0\right)}{f_{Y, D}\left(y_{0}, 0 \mid Z=0\right)} \text { for all } y=\left(y_{0}, y_{1}\right) \in \mathcal{L},
$$

and

$$
f_{Y, D}\left(y_{1}, 1 \mid Z=0\right)>0, \quad f_{Y, D}\left(y_{0}, 0 \mid Z=1\right)>0, \quad \text { for all } y=\left(y_{0}, y_{1}\right) \in \mathcal{L}
$$

The proof that these are sufficient conditions is given in the appendix, and below we discuss the economic plausibility of these conditions.

Comment 3.2 (Plausibility of (3.15) and (3.16) ). The condition (3.16) seems quite mild, so we focus on (3.15). We can illustrate (3.15) by considering the problem of evaluating a training program where $Y$ 's are earnings, $D$ 's $\in\{0,1\}$ are training states, and $Z$ 's $\in\{0,1\}$ are offers of training service. Condition (3.15) may be interpreted as a monotone likelihood ratio condition. That is, the instrument $Z$ should have a monotonic impact on the likelihood ratio specified in (3.15). This monotonicity may be a weak condition in some contexts and a strong condition in others. For instance, if $\mathcal{L}$ is a cube $q+C$, then this condition may be considered relatively strong. On the other hand, if we impose monotonicity of the training impact on earning quantiles, so that $q(0) \leqslant q(1)$, i.e. $q \in \mathcal{L}=$ $(q+C) \cap H$, then condition (3.15) would be trivially satisfied in many empirical settings. Indeed, it would suffice that the instrument $Z$, the offer of training services, increases the relative joint likelihood of receiving higher earnings and receiving the training service. In many instances, we also have $P[D=1 \mid Z=0]=0$; e.g. those not offered training services do not receive that training. When $P[D=1 \mid Z=0]=0$, the right-hand side of (3.15) 
equals 0 which makes the identification condition (3.15) satisfied trivially even for the less convenient parameter sets such as $\mathcal{L}=q+C$.

3.2. Identification with Multiple Points of Support. We generalize the result of Theorem 2 to more general discrete treatments with discrete instruments. Consider the case when $D$ has the support $\{1, \ldots, l\}$ and $Z$ has the support $\{1, \ldots, r\}(l \leqslant r<\infty)$. Note that function $q(\cdot)$ can be represented by a vector $q=(q(1), \ldots, q(l))^{\prime} \in \mathbb{R}^{l}$. Under the conditions of Theorem 1 , there is at least one function $q(d)$ that solves $P[Y \leqslant q(D) \mid Z]=$ $\tau$ a.s. Therefore, for vectors of the form $y=\left(y_{1}, \ldots, y_{l}\right)^{\prime}$ and the vector of moment equations

$$
\Pi(y)=\left(P\left[Y \leqslant y_{D} \mid Z=z\right]-\tau, \quad z=1, \ldots, r\right)^{\prime},
$$

where $y_{D}:=\sum_{d} 1[D=d] \cdot y_{d}$, the model is identified if $y=q$ uniquely solves $\Pi(y)=0$.

We define matrix $\partial \Pi(y)$ as the $r \times l$ matrix with $(d, z)$ element given by $f_{Y}\left(y_{d} \mid D=d, Z=\right.$ z) $P[D=d \mid Z=z]$ where $z=1, \ldots, r$ and $d=1, \ldots, l$. We require this to be the Jacobian matrix of the map $y \mapsto \Pi(y)$ and impose full-rank-type conditions on submatrices of this Jacobian. To this end, let $m$ denote any permutation of $l$ distinct integers from $\{1, \ldots, r\}$, called $l$-permutations, and $\mathcal{M}$ be a collection of all such permutations. Let $\Pi_{m}:=\left(\Pi_{j}\right)_{j \in m}$, which maps $\mathbb{R}^{l}$ to $\mathbb{R}^{l}$, be a subvector of $\Pi$ formed by selecting $j$-th elements of $\Pi$ according to their order in $m$ Let $\partial \Pi_{m}$ denote the corresponding $l \times l$ Jacobian matrix of $\Pi_{m}$. The following theorem generalizes Theorem 2 .

Theorem 3 (Identification for Discrete D). Suppose $\Pi(q)=0$, the support of $D$ is $\{1, \ldots, l\}$ and of $Z$ is $\{1, \ldots, r\}$. Assume that the conditional density $f_{Y}(y \mid D=d, Z=z)$ exists for each $y \in \mathbb{R}$, and $(d, z) \in\{1, \ldots, l\} \times\{1, \ldots, r\}$. (i) (Local) Suppose the Jacobian $\partial \Pi(y)$ defined above is continuous and has rank $l$ at $y=q$. Then the $\tau$-quantiles of potential outcomes, $q$, are identified in the region $\mathcal{L}$ given by a sufficiently small open neighborhood of $q$ in $\mathbb{R}^{l}$. (ii) (Global) Assume that region $\mathcal{L}$ contains $q$ and can be covered by a finite number of compact convex l-dimensional polytopes $\left\{\mathcal{L}_{j}\right\}$, each containing $q$ and having the following properties: For each $j$ there is an l-permutation $m(j) \in \mathcal{M}$, such that $\partial \Pi_{m(j)}$ is the $C^{1}$ Jacobian of $\Pi_{m(j)}: \mathcal{L}_{j} \rightarrow \mathbb{R}^{l}$, and for each $y \in \mathcal{L}_{j}$ and each subspace $L \subset \mathbb{R}^{l}$ spanned by a face of $\mathcal{L}_{j}$ that includes $y$, the linear map

$$
\operatorname{proj}_{L} \circ \partial \prod_{m(j)}(y): L \mapsto L
$$

has a positive determinant. Then $q$ is identified in $\mathcal{L}$.

\footnotetext{
${ }^{11}$ Note that this formulation allows reordering elements of $\Pi$ which may be needed to achieve the required positive determinant condition as discussed in the binary case.
} 
We note that in the theorem existence of the conditional density $f_{Y}(y \mid D=d, Z=z)$ is only required for $(d, z)$ in the support of $(D, Z)$. This density can be defined to take on an arbitrary value for $(d, z)$ outside the support. The first result is a simple local identification condition provided to fix ideas. The second result is a global identification condition based on Global Univalence Theorem 1 of Mas-Colell (1979). This result complements a similar result given in Chernozhukov and Hansen (2005) based on Global Univalence Theorem 2 of Mas-Colell (1979). The positive determinant condition requires the impact of instrument $Z$ on the joint distribution of $(Y, D)$ to be sufficiently rich.

Comment 3.3 (An Alternative Sufficient Condition). Here we recall an alternative sufficient condition from Chernozhukov and Hansen (2005), which is based on the Global Univalence Theorem 2 of Mas-Colell (1979). Assume that region $\mathcal{L}$ contains $q$ and can be covered by a finite number of compact convex $l$-dimensional sets $\left\{\mathcal{L}_{j}\right\}$, each containing $q$ and having the following properties: (i) For each $j$, there is a permutation $m(j) \in \mathcal{M}$ such that $\partial \Pi_{m(j)}$ is $C^{1}$ Jacobian of $\Pi_{m(j)}: \mathcal{L}_{j} \rightarrow \mathbb{R}^{l} ;$ (ii) for each $y \in \mathcal{L}_{j}$,

$$
\operatorname{det}\left[\partial \prod_{m(j)}(y)\right]>0 ;
$$

(iii) $\mathcal{L}_{j}$ possesses a $C^{1}$-smooth boundary $\partial \mathcal{L}_{j}$; and (iv) for each $y \in \partial \mathcal{L}_{j}, l^{\prime}\left(\partial \prod_{m(j)}(y)+\right.$ $\left.\partial \Pi_{m(j)}(y)^{\prime}\right) l>0$ for each $l \in T(y): l \neq 0$ where $T(y)$ is the subspace tangent to $\mathcal{L}_{j}$ at point $y$. Then $q$ is identified in $\mathcal{L}$. This condition seems to require slightly stronger conditions on the boundary than the condition used in Theorem 3. The advantage of the conditions from Chernozhukov and Hansen (2005) is that they more transparently convey the full-rank nature of the conditions imposed.

3.3. Identification with general D. Finally we consider conditions for point identification in the case of more general $D$ and $Z$ that may take on a continuum of values. We let $d$ denote elements in the support of $D$ and $z$ denote elements in the support of $Z$. Without loss of much generality, we restrict attention to the case where both $Y$ and $D$ have bounded support. We require the parameter space $\mathcal{L}$ to be a collection of bounded (measurable) functions $m: \mathbb{R}^{k} \mapsto \mathbb{R}$ containing $q(\cdot)$. We say that $q(\cdot)$ such that $P[Y \leqslant q(D) \mid Z]=\tau$ a.s. is identified in $\mathcal{L}$ if for any other $m(\cdot) \in \mathcal{L}$ such that $P[Y \leqslant m(D) \mid Z]=\tau$ a.s., $m(D)=q(D)$ a.s. Below, we use $\|\cdot\|_{p, P}$ to denote the $L^{p}(P)$ norm.

Theorem 4 (Identification with General $D$ ). Suppose that $P[Y \leqslant q(D) \mid Z]=\tau$ a.s. and both $Y$ and $D$ have bounded support. Consider a parameter space $\mathcal{L}$ which is a collection of bounded (measurable) functions $m: \mathbb{R}^{k} \mapsto \mathbb{R}$ containing $q(\cdot)$. Assume that for $\epsilon:=$ 
$Y-q(D)$ the conditional density $f_{\epsilon}(e \mid D, Z)$ exists for each $e \in \mathbb{R}$, a.s. (i) (Global) Suppose that for each $\Delta(d):=m(d)-q(d)$ with $m(\cdot) \in \mathcal{L}, \omega_{\Delta}(D, Z):=\int_{0}^{1} f_{\epsilon}(\delta \Delta(D) \mid D, Z) d \delta>0$ a.s. and

$$
E\left[\Delta(D) \cdot \omega_{\Delta}(D, Z) \mid Z\right]=0 \text { a.s. } \Rightarrow \Delta(D)=0 \text { a.s. }
$$

Then $q(\cdot)$ is identified in $\mathcal{L}$. (ii) (Local) Suppose that $\omega_{0}(D, Z):=f_{\epsilon}(0 \mid D, Z)>0$ a.s. and for each $\Delta(d):=m(d)-q(d)$ with $m(\cdot) \in \mathcal{L}$,

$$
E\left[\Delta(D) \cdot \omega_{0}(D, Z) \mid Z\right]=0 \text { a.s. } \Rightarrow \Delta(D)=0 \text { a.s. },
$$

and, for some $0 \leqslant \eta<1$ and $1 \leqslant p$,

$$
\left\|E\left[\Delta(D) \cdot\left\{\omega_{\Delta}(D, Z)-\omega_{0}(D, Z)\right\} \mid Z\right]\right\|_{p, P} \leqslant \eta\left\|E\left[\Delta(D) \cdot \omega_{0}(D, Z) \mid Z\right]\right\|_{p, P} .
$$

Then $q(\cdot)$ is identified in $\mathcal{L}$.

Condition (i), mentioned in Chernozhukov and Hansen (2005), states a non-linear bounded completeness condition for global identification. The condition (3.20) required is not primitive, but it highlights a useful link with the linear bounded completeness condition: $E[\Delta(D) \mid Z]=0$ a.s. $\Rightarrow \Delta(D)=0$ a.s. used by Newey and Powell (2003). The latter condition is needed for identification in the mean IV model $E[Y-q(D) \mid Z]=0$ under the assumption of a bounded structural function $q$. The latter condition is known to be quite weak, as shown in D'Haultfoeuille (2011), and there are many primitive sufficient conditions that imply this condition. Andrews (2011) shows that linear completeness is generic under some conditions. Although condition (3.20) is not primitive, it is not vacuous either since the previous theorems provide primitive conditions for its validity. The local identification condition (ii), obtained by Chen, Chernozhukov, Lee, and Newey (2011), provides yet another sufficient condition for condition (i). The result (ii) replaces the nonlinear completeness condition (3.20) by the linear completeness condition (3.21) which is easier to check. The result (ii) also implicitly requires that the set $\mathcal{L}$ is a sufficiently small neighborhood of $q$ and that functional deviations $m(\cdot)-q(\cdot)$ and the conditional density $f_{\epsilon}(\cdot \mid D, Z)$ are sufficiently smooth. This is explained in detail in Chen, Chernozhukov, Lee, and Newey (2011) where further primitive smoothness and completeness conditions are also provided.

\section{Other Approaches to Quantile Models with Endogeneity}

There are, of course, other sets of modeling assumptions that one could employ to build a quantile model with endogeneity. In this section, we briefly outline two other 
approaches that have been taken in the literature. The first, due to Abadie, Angrist, and Imbens (2002), extends the local average treatment effect (LATE) framework of Imbens and Angrist (1994) to quantile treatment effects. The second, considered in Imbens and Newey (2009) and Lee (2007), uses a triangular structure to obtain identification.

\subsection{Local Quantile Treatment Effects with Binary Treatment and Instrument.}

In fundamental work, Abadie, Angrist, and Imbens (2002) develop an approach to estimating quantile treatment effects within the LATE framework of Imbens and Angrist (1994) in the case where both the instrument and treatment variable are binary. The use of the LATE framework makes this approach appealing as many applied researchers are familiar with LATE and the conditions that allow identification and consistent estimation of this quantity. Importantly, the extension proceeds under exactly the same monotonicity requirement as needed for LATE.

Specifically, Abadie, Angrist, and Imbens (2002) show that the QTE for a subpopulation is identified if

1. (Independence) the instrument $Z$ is independent of the potential outcome errors, $\left\{U_{d}\right\}$, and the errors in the selection equation, $V$;

2. (Monotonicity) $P\left(D_{1} \geq D_{0} \mid X\right)=1$ where $D_{1}$ is the treatment state of an individual when $Z=1$ and $D_{0}$ is defined similarly, holds;

3. and other standard conditions are met.

The subpopulation for whom the QTE is identified is the set of "compliers," those individuals with $D_{1}>D_{0}$. In other words, the compliers are the set of individuals whose treatment is altered by switching the instrument from zero to one. Monotonicity is key in this framework. The monotonicity condition rules out "defiers," individuals who would receive treatment in the absence of the intervention represented by the instrument but would not receive treatment if placed into the treatment group. The effects for individuals who would always receive treatment or never receive treatment regardless of the value of the instrument are unidentified.

Looking at these conditions, we see that the model of Abadie, Angrist, and Imbens (2002) replaces the monotonicity assumption (A1), the independence assumption (A2), and the similarity assumption (A4) with a different type of monotonicity and a stronger independence assumption and identifies a different quantity: the QTE for compliers. The LATE-style approach has not yet been extended beyond cases with a binary treatment and 
a single binary instrument while the instrumental variable quantile model of Chernozhukov and Hansen (2005) applies to any endogenous variables and instruments. Note that neither set of conditions nests the other, and neither framework is more general than the other. Thus, the frameworks are best viewed as complements, providing two sets of conditions that can be considered when thinking about a strategy for estimating heterogeneous treatment effects.

Of course, the two sets of conditions may be mutually compatible. One such case is discussed in Chernozhukov and Hansen (2004). In this example, the pattern of results obtained from the two estimators is quite similar, and the difference between the estimates appears small relative to sampling variation. Further exploration of these two approaches and their similarities and differences may be interesting to consider.

\subsection{Instrumental Variables Quantile Regression in Triangular Systems. An-} other compelling framework is based on assuming a triangular structure as in Imbens and Newey (2009). See also Chesher (2003), Koenker and Ma (2006), and Lee (2007) for related models and results. The triangular model takes the form of a triangular system of equations

$$
\begin{aligned}
& Y=g(D, \epsilon), \\
& D=h(Z, \eta),
\end{aligned}
$$

where $Y$ is the outcome, $D$ is a continuous scalar endogenous variable, $\epsilon$ is a vector of disturbances, $Z$ is a vector of instruments with a continuous component, $\eta$ is a scalar reduced form error, and we ignore other covariates for simplicity. It is important to note that the triangular system generally rules out simultaneous equations which typically have that the reduced form relating $D$ to $Z$ depends on a vector of disturbances. For example, in a supply and demand system, the reduced form for both price and quantity will generally depend on the unobservables from both the supply equation and the demand equation. Outside of $\eta$ being a scalar, the key conditions that allow identification of quantile effects in the triangular system are

1. (Monotonicity) The function $\eta \mapsto h(Z, \eta)$ is strictly increasing in $\eta$, and

2. (Independence) $D$ and $\epsilon$ are independent conditional on $V$ for some observable or estimable $V$.

The variable $V$ is thus the "control function" conditional on which changes in $D$ may be taken as causal. Imbens and Newey (2009) use $V=F_{D \mid Z}(d, z)=F_{\eta}(\eta)$, where $F_{\eta}(\cdot)$ 
represents the CDF of $\eta$, as the control function and show that this variable satisfies the independence condition under the additional condition that $(\epsilon, \eta)$ is independent of $Z$. They show that one may use $D=h(Z, \eta)$ to identify $V$ under the assumed monotonicity of $h(Z, \eta)$ in $\eta$. Using $V$ obtained in this first step, one may then construct the distribution of $Y \mid D, V$. Then integrating over the distribution of $V$ and using iterated expectations, one has

$$
\begin{aligned}
\int F_{Y \mid D, V}(y \mid d, v) F_{V}(d v) & =\int 1(g(d, \epsilon) \leq y) F_{\epsilon}(d \epsilon) \\
& =\operatorname{Pr}(g(d, \epsilon) \leq y):=G(y, d) .
\end{aligned}
$$

It then follows that the $\tau^{\text {th }}$ quantile of $Y_{d}$ is $G^{-1}(\tau, d)$.

As with the framework of Abadie, Angrist, and Imbens (2002), the triangular model under the conditions given above is neither more nor less general than the model of Chernozhukov and Hansen (2005). The key difference between the approaches is that Chernozhukov and Hansen (2005) uses an essentially unrestricted reduced form but requires monotonicity and a scalar disturbance in the structural equation. The triangular system on the other hand relies on monotonicity of the reduced form in a scalar disturbance. In addition, the triangular system, as developed in Imbens and Newey (2009), requires a more stringent independence condition in that the instruments need to be independent of both the structural disturbances and the reduced form disturbance. That the approaches impose structure on different parts of the model makes them complementary with a researcher's choice between the two being dictated by whether it is more natural to impose restrictions on the structural function or the reduced form in a given application.

The triangular model and the model of Chernozhukov and Hansen (2005) can be made compatible by imposing the conditions from the triangular model on the reduced form and the conditions from Chernozhukov and Hansen (2005) on the structural model. Torgovitsky (2012) considers identification and estimation when both sets of conditions are imposed and shows that the requirements on the instruments may be substantially relaxed relative to Chernozhukov and Hansen (2005) or Imbens and Newey (2009) in this case.

\section{Estimation And InFEREnCE}

In the previous sections, we have outlined results that are useful for identifying quantile treatment effects and structural functions that are monotonic in a scalar unobservable. In the following, we briefly review the literature on estimation and inference. We focus 
on estimation of the model of Chernozhukov and Hansen (2005) presented in Section 2 using the moment conditions derived in Theorem 1. For estimation of the triangular model, see Imbens and Newey (2009) for nonparametric estimation and Lee (2007) for a semiparametric approach. Abadie, Angrist, and Imbens (2002) provides results for estimating the QTE for compliers within the LATE-style framework. Also, we only review approaches for estimating parametric quantile functions: $q(D, X, \tau)=g(D, X, \tau ; \theta)$ for $\theta \in \Theta \subset \mathbb{R}^{m}$. Horowitz and Lee (2007) and Gagliardini and Scaillet (2012) present nonparametric estimation and inference results for the IVQT model using condition (2.3).

There are two practical issues that make estimation and inference based on condition (2.3) challenging. The first is that the sample analog to condition (2.3) is non-smooth, and the GMM objective function that would be formed by using (2.3) as the moment conditions is also generically non-convex, even for linear quantile models. The second problem is that the model may suffer from weak identification as in the standard linear IV model; Stock, Wright, and Yogo (2002) provides a useful introductory survey to weak identification and related inference methods in the linear IV model. In the quantile case, the problem of weak identification is more subtle than in the linear model in that some quantiles may be weakly identified while others may be strongly identified. The relevant object for defining the strength of identification of a given quantile is the covariance between $D$ and $Z$ weighted by the conditional density function of the unobservable at the given quantile. See Chernozhukov and Hansen (2008) for a formal definition of this object and related discussion.

While the non-smoothness and non-convexity of the GMM criterion complicates optimization, it does not render the approach infeasible, especially when the dimension of $D$ and $X$ is not too large. Abadie (1997) considered this approach for estimating an income model and provides further discussion. One could also estimate the model parameters using the Markov Chain Monte Carlo (MCMC) approach of Chernozhukov and Hong (2003). This approach bypasses the need for optimization, instead relying on sampling and averaging to estimate model parameters. Note that this approach is not a cure-all since MCMC requires careful tuning in applications. It is also worth noting that standard samplers may perform poorly in even simple linear instrumental variables models when identification is not strong; see Hoogerheide, Kaashoek, and van Dijk (2007). In an approach related to optimizing the GMM criterion function directly, Sakata (2007) proposes estimating the parameters of an instrumental variables quantile model by optimizing a different non-smooth, non-convex criterion function. 
To partially circumvent the numerical problems in optimizing the full GMM criterion, Chernozhukov and Hansen (2006) suggest a different procedure termed the inverse quantile regression for the linear quantile model $q(D, X, \tau)=D^{\prime} \alpha(\tau)+X^{\prime} \beta(\tau)$. The basic intuition for the inverse quantile regression comes from the observation that if one knew the true value of the coefficient on $D, \alpha(\tau)$, the $\tau^{\text {th }}$ quantile regression of $Y-D^{\prime} \alpha(\tau)$ onto $X$ and $Z$ would yield zero coefficients on the instruments $Z$. This observation allows one to effectively concentrate $\beta(\tau)$ out of the problem and leaves a non-smooth, non-convex optimization problem over only the parameters $\alpha(\tau)$. Since $D$ is low-dimensional in many applications, one can usually solve this optimization problem using highly robust optimization procedures such as a grid-search.

Algorithmically, the inverse quantile regression estimates for a given probability index $\tau$ of interest can be obtained as follows using a grid search over $\alpha(\tau)$ :

1. Define a suitable set of values $\left\{\alpha_{j}, j=1, \ldots, J\right\}$, and estimate the coefficients $\beta\left(\alpha_{j}, \tau\right)$ and $\gamma\left(\alpha_{j}, \tau\right)$ from the model $Y-D^{\prime} \alpha_{j}=X^{\prime} \beta\left(\alpha_{j}, \tau\right)+Z^{\prime} \gamma\left(\alpha_{j}, \tau\right)+\epsilon$ by running the ordinary $\tau$-quantile regression of $Y-D^{\prime} \alpha_{j}$ on $X$ and $Z$. Call the estimated coefficients $\widehat{\beta}\left(\alpha_{j}, \tau\right)$ and $\widehat{\gamma}\left(\alpha_{j}, \tau\right)$.

2. Save the inverse of the variance-covariance matrix of $\widehat{\gamma}\left(\alpha_{j}, \tau\right)$, which is readily available in any common implementation of the ordinary QR. Denote this variance-covariance matrix $\widehat{A}\left(\alpha_{j}, \tau\right)$. Form $W_{n}\left(\alpha_{j}, \tau\right)=\widehat{\gamma}\left(\alpha_{j}, \tau\right)^{\prime} \widehat{A}\left(\alpha_{j}, \tau\right)^{-1} \widehat{\gamma}\left(\alpha_{j}, \tau\right)$. Note $W_{n}\left(\alpha_{j}\right)$ is the Wald statistic for testing $\gamma\left(\alpha_{j}, \tau\right)=0$.

3. Choose $\widehat{\alpha}(\tau)$ as a value among $\left\{\alpha_{j}, j=1, \ldots, J\right\}$ that minimizes $W_{n}(\alpha, \tau)$. The estimate of $\beta(\tau)$ is then given by $\widehat{\beta}(\widehat{\alpha}(\tau), \tau)$.

Chernozhukov and Hansen (2006) and Chernozhukov and Hansen (2008) provide conditions under which the resulting estimator for $\alpha(\tau)$ and $\beta(\tau)$ is consistent and asymptotically normal and provide a consistent variance estimator. Marmer and Sakata (2012) provide a similar multi-step algorithm that circumvents the same numeric problems using the objective function of Sakata (2007).

The good behavior of the asymptotic approximations obtained in Chernozhukov and Hansen (2006) and Chernozhukov and Hansen (2008) rely on strong identification of the model parameters just as in the linear IV case. Intuitively, strong identification for a quantile of interest requires that a particular density-weighted covariation matrix between $D$ and $Z$ is not local to being rank deficient and that the impact of $Z$ is rich enough to guarantee that the moment equations have a unique solution. The first condition is 
analogous to the usual full rank condition in linear IV analysis, and the second condition is required because of the nonlinearity of the problem. Checking these conditions in practice may be difficult, and it is therefore useful to have inference procedures that are robust to violations of these conditions.

Fortunately, there are several inference procedures that remain valid under weak identification. A nice feature of the algorithm defined for estimating $\alpha(\tau)$ above is that it produces a weak-identification-robust inference procedure naturally as a byproduct. Chernozhukov and Hansen (2008) show that the Wald statistic, $W_{n}(\alpha, \tau)$ converges in

distribution to $\chi_{\operatorname{dim}(Z)}^{2}$ under the null that $\alpha=\alpha_{0}$ where we let $\alpha_{0}$ denote the true value of $\alpha(\tau)$ without needing either of the conditions discussed in the preceding paragraph. Thus a valid $(1-p) \%$ confidence region for $\alpha(\tau)$ may be constructed as the set:

$$
\left\{\alpha: W_{n}(\alpha, \tau) \leqslant c_{1-p}\right\}
$$

where $c_{1-p}$ is such that $\operatorname{Pr}\left(\chi_{\operatorname{dim}(Z)}^{2}>c_{1-p}\right)=p$, and the set is approximated numerically by considering $\alpha$ 's in the grid $\left\{\alpha_{j}, j=1, \ldots, J\right\}$. Chernozhukov and Hansen (2008) show that confidence region in equation (5.23) is valid when the model parameters are strongly identified and remains valid when the model is weakly identified or even unidentified. Marmer and Sakata (2012) provide a similar procedure and result for their procedure as well. Jun (2008) provides yet a different approach to performing weak-identificationrobust inference in models defined by conditions (2.3). Finally, Chernozhukov, Hansen, and Jansson (2009) show that one can form statistics for inference about the entire parameter vector $\theta$ that are condtionally pivotal in finite-samples for models defined by quantile restrictions such as (2.3). Since the statistics do not depend on unknown nuisance parameters in finite samples, the exact distributions of these statistics can be calculated and inference can proceed without relying on asymptotic approximations or statements about the strength of identification. The distributions produced in Chernozhukov, Hansen, and Jansson (2009) are not standard and so must be calculated by simulation.

\section{Conclusion and Directions for Future Research}

In this paper, we have reviewed approaches for building quantile models in the presence of endogeneity, focusing on conditions that can be used for identification. We have also briefly reviewed some of the practical issues that arise in estimation of instrumental variables quantile models and approaches to dealing with these issues. The models and 
estimation strategies outlined and cited in this review have already seen use in empirical economics where they have mostly been used for their ability to uncover interesting distributional effects. In this review, we have also noted that the identification strategy employed in this paper can be used to uncover structural objects even if quantile effects are not the chief objects of interest as in Berry and Haile (2010).

While the results reviewed in this paper are useful in a variety of contexts, there remain interesting areas for research in quantile models with endogeneity. In some applications, features of the conditional distribution are not the chief objects of interest and researchers are interested in effects of treatments on unconditional quantiles. Given the set of conditional quantiles, such unconditional effects may be uncovered. In recent work, Froelich and Melly (2008) propose a different approach, related to Abadie, Angrist, and Imbens (2002), to estimating structural effects of endogenous variables on unconditional quantiles directly. It would also be interesting to think about quantile-like quantities for multivariate outcomes with endogenous covariates. The results reviewed in this paper offer one possible approach for quantile modeling with endogeneity, but there remain many interesting directions and other approaches to be explored in further research.

\section{Appendix A. Proofs}

A.1. Proof of Theorem 1. Conditioning on $X=x$ is suppressed. For $P$ almost every value $z$ of $Z$,

$$
\begin{aligned}
P\left[U_{D} \leqslant \tau \mid Z=z\right] & \stackrel{(1)}{=} \int P\left[U_{D} \leqslant \tau \mid Z=z, V=v\right] d P[V=v \mid Z=z] \\
& \stackrel{(2)}{=} \int P\left[U_{\delta(z, v)} \leqslant \tau \mid Z=z, V=v\right] d P[V=v \mid Z=z] \\
& \stackrel{(3)}{=} \int P\left[U_{0} \leqslant \tau \mid Z=z, V=v\right] d P[V=v \mid Z=z] \\
& \stackrel{(4)}{=} P\left[U_{0} \leqslant \tau \mid Z=z\right] \stackrel{(5)}{=} \tau .
\end{aligned}
$$

Equality (1) is by definition. Equality (2) is by the representation A3. Equality (3) is by the similarity assumption $\mathrm{A} 4$ and representation A3: Conditional on $(V=v, Z=z)$, $D=\delta(z, v)$ is a constant, so that by $\mathrm{A} 4, U_{\delta(z, v)}$ has the same distribution as $U_{0}$, where " 0 " denotes any fixed value of $D$. Equality (4) is by definition, and equality (5) is by the independence assumption A2. This shows the first result. 
The second result follows from the first and the equivalence of the events $\{q(D, U) \leqslant$ $q(D, \tau)\}=\{U \leqslant \tau\}$ under $u \mapsto q(d, u)$ strictly increasing for each $d$ on the domain $[0,1]$. To show the third result we note that

$$
\{U \in I\} \subseteq\{\{u: q(D, u)=q(D, U)\} \cap I \neq \oslash\}
$$

Since $Y=q(D, U)$, the latter event is equivalent to the event $\{Y \in q(D, I)\}$, where $q(D, I)$ denotes the image of $I$ under the mapping $u \mapsto q(D, u)$. The third result then follows from the first result.

A.2. Proof of Theorems $\mathbf{2}$ and 3 . The local identification results follow by a standard argument, introduced in Rothenberg (1971), which we omit for brevity. The global identification result is obtained as follows. By assumption $q \in \mathcal{L}$. Hence, we need to check whether $y=q$ is the only solution to $\Pi(y)=0$ over $\mathcal{L}$. Consider a covering set $\mathcal{L}_{j}$ and the $l$-permutation $m(j)$ corresponding to it, as defined in the theorem. By assumption $\Pi_{m(j)}(q)=0$. By assumption $q \in \mathcal{L}_{j}$. The stated rank conditions, compactness, and convexity of the polytope $\mathcal{L}_{j}$ imply that the mapping $y \rightarrow \Pi_{m(j)}(y)$, which maps $\mathcal{L}_{j} \subset \mathbb{R}^{l}$ to $\mathbb{R}^{l}$, is a homeomorphism (one-to-one) between $\mathcal{L}_{j}$ and $\Pi_{m(j)}\left(\mathcal{L}_{j}\right)$ by the global univalence theorem, Theorem 1 of Mas-Colell (1979). Thus, $y=q$ is the unique solution of $\Pi_{m(j)}(y)=0$ over $\mathcal{L}_{j}$. Since this argument applies to every $j$ and $\left\{\mathcal{L}_{j}\right\}$ cover $\mathcal{L}$, it follows that $y=q$ is the unique solution of $\Pi(y)=0$ over $\mathcal{L}$.

A.3. Proof of Theorem 4. We have that $q$ solves $P[Y \leqslant q(D) \mid Z]=\tau$ a.s., and $q \in \mathcal{L}$ by assumption. Hence we need to check whether $q$ is the only solution to $P[Y \leqslant q(D) \mid Z]=\tau$ a.s. in $\mathcal{L}$. Suppose there is $m \in \mathcal{L}$ such that $P[Y \leqslant m(D) \mid Z]=\tau$ a.s. Define $\Delta(d):=$ $m(d)-q(d)$, and write

$$
\begin{aligned}
P[Y \leqslant m(D) \mid Z] & -P[Y \leqslant q(D) \mid Z] \stackrel{(1)}{=} E\left[E\left[\int_{0}^{1} f_{\epsilon}(\delta \Delta(D) \mid D, Z) \Delta(D) d \delta \mid D, Z\right] \mid Z\right] \\
& \stackrel{(2)}{=} E\left[\int_{0}^{1} f_{\epsilon}(\delta \Delta(D) \mid D, Z) \Delta(D) d \delta \mid Z\right] \\
& \stackrel{(3)}{=} E\left[\Delta(D) \cdot \omega_{\Delta}(D, Z) \mid Z\right] .
\end{aligned}
$$

Noting that (1) follows by the fundamental theorem of calculus, (2) by the law of iterated expectations, and (3) by linearity of the Lebesgue integral. For uniqueness we need that $(\underline{A .25})=0$ a.s. $\Rightarrow \Delta(D)=0$ a.s., which is assumed. The result (i) follows.

Result (ii) is immediate from (i) by the triangle inequality for $\|\cdot\|_{p, P}$. 
IVQR

A.4. Proof of Sufficiency of (3.15) and (3.16). Here show that (3.15) and (3.16) are sufficient for identification over the parameter space $\mathcal{L}=(q+C) \cap H$. Note that in general $\mathcal{L}$ has at most up to five edges: the left and the right edges, parallel to each other, the top and the bottom edges, also parallel to each other, and the edge generated by the intersection of $\mathcal{L}$ with the 45 degree line. Let $e_{1}$ and $e_{2}$ be coordinate vectors in $\mathbb{R}^{2}$, and let $L_{k}$ denote the various subspaces spanned by faces of $\mathcal{L}$ containing $y$. In particular, we have that $L_{1}:=\mathbb{R}^{2}$ for all $y$ in the two-dimensional face $F_{1}:=\mathcal{L}, L_{2}:=\operatorname{span}\left(e_{2}\right)$ for all $y$ in the one-dimensional faces given by the left and the right edge of $\mathcal{L}$, denoted both by $F_{2}$, $L_{3}:=\operatorname{span}\left(e_{1}\right)$ for all $y$ in the two-dimensional faces given by the top and bottom edges of $\mathcal{L}$, denoted both by $F_{3}$, and $L_{4}=\operatorname{span}\left(e_{1}+e_{2}\right)$ for all $y$ in the one-dimensional face $F_{4}$ given by the edge generated by the intersection of $\mathcal{L}$ with the 45 degree line. The subspaces spanned by vertices, which are zero-dimensional faces of $\mathcal{L}$, are null spaces; so we do not need to consider them. We compute the projections of the Jacobian map onto these subspaces: $\operatorname{proj}_{L_{1}} \circ \partial \Pi(y)[l]=\partial \Pi(y) l, \operatorname{proj}_{L_{2}} \circ \partial \Pi(y)[l]=f_{Y, D}\left(y_{0}, 0 \mid Z=0\right) l, \operatorname{proj}_{L_{3}} \circ$ $\partial \Pi(y)[l]=f_{Y, D}\left(y_{1}, 1 \mid Z=1\right) l, \operatorname{proj}_{L_{4}} \circ \partial \Pi(y)[l]=\left\{\left[f_{Y, D}\left(y_{1}, 1 \mid Z=1\right)+f_{Y, D}\left(y_{0}, 0 \mid Z=\right.\right.\right.$ $\left.\left.1)+f_{Y, D}\left(y_{1}, 1 \mid Z=0\right)+f_{Y, D}\left(y_{0}, 0 \mid Z=0\right)\right] / 2\right\} l$, for $y \in F_{k}$ and $l \in L_{k}$ in each of the cases. We then compute the corresponding determinants of the maps

$$
\operatorname{proj}_{L_{k}} \circ \partial \Pi(y): L_{k} \rightarrow L_{k}
$$

where determinants are computed with respect to the coordinate systems of $L_{k}$, as $\operatorname{det}[\partial \Pi(y)]$ for $k=1, f_{Y, D}\left(y_{0}, 0 \mid Z=0\right)$ for $k=2, f_{Y, D}\left(y_{1}, 1 \mid Z=1\right)$ for $k=3,\left[f_{Y, D}\left(y_{1}, 1 \mid Z=\right.\right.$ $\left.1)+f_{Y, D}\left(y_{0}, 0 \mid Z=1\right)+f_{Y, D}\left(y_{1}, 1 \mid Z=0\right)+f_{Y, D}\left(y_{0}, 0 \mid Z=0\right)\right] / 2$ for $k=4$. Theorem 2 requires that these determinants are positive for values of $y \in F_{k}$. This condition is implied by the simpler conditions (3.15) and (3.16). For the case of $\mathcal{L}=q+C$, verification is analogous except that we do not need to consider $L_{4}$. Thus, the positive determinant condition of Theorem 2 is implied by the conditions (3.15) and (3.16) for $\mathcal{L}=q+C$ as well.

\section{REFERENCES}

Abadie, A. (1997): "Changes in Spanish Labor Income Structure During the 1980s: A Quantile Regression Approach," Investigaciones Económicas, 21(2), 253-272.

Abadie, A., J. Angrist, And G. Imbens (2002): "Instrumental variables estimates of the effect of subsidized training on the quantiles of trainee earnings," Econometrica, 70(1), 91-117.

Andrews, D. W. K. (2011): "Examples of $L^{2}$-complete and boundedly-complete distributions," Cowles Foundation Discussion Paper No. 1801. 
Andrews, D. W. K., And X. Shi (2013): "Inference Based on Conditional Moment Inequalities," forthcoming in Econometrica.

Autor, D. H., S. N. Houseman, And S. P. Kerr (2012): "The Effect of Work First Job Placements on the Distribution of Earnings: An Instrumental Variable Quantile Regression Approach," NBER Working Paper No. 17972.

Beresteanu, A., I. Molchanov, And F. Molinari (2011): "Sharp Identification Regions in Models With Convex Moment Predictions," Econometrica, 79(6), 1785-1821.

Berry, S. T., AND P. A. Haile (2010): "Identification in Differentiated Products Markets Using Market Level Data," Cowles Foundation Discussion Paper No. 1744.

Chen, X., V. Chernozhukov, S. Lee, And W. K. Newey (2011): "Local identification of nonparametric and semiparametric models," arXiv:1105.3007v1 [math.ST].

Chen, X., O. Linton, And I. V. Keilegom (2003): "Estimation of semiparametric models when the criterion function is not smooth," Econometrica, 71, 1591-1608.

Chernozhukov, V., And C. Hansen (2004): "The Effects of 401(k) Participation on the Wealth Distribution: An Instrumental Quantile Regression Analysis," Review of Economics and Statistics, 86(3), 735-751.

- (2005): "An IV Model of Quantile Treatment Effects," Econometrica, 73(1), 245-262.

(2006): "Instrumental Quantile Regression Inference for Structural and Treatment Effect Models," Journal of Econometrics, 132(2), 491-525.

(2008): "Instrumental variable quantile regression: A robust inference approach," Journal of Econometrics, 142(1), 379-398.

Chernozhukov, V., C. Hansen, and M. Jansson (2009): "Finite sample inference for quantile regression models," Journal of Econometrics, 152(2), 93-103.

Chernozhukov, V., AND H. Hong (2003): "An MCMC Approach to Classical Estimation," Journal of Econometrics, 115, 293-346.

Chernozhukov, V., G. W. Imbens, and W. K. Newey (2007): "Instrumental variable estimation of nonseparable models," Journal of Econometrics, 139(1), 4-14.

Chernozhukov, V., S. Lee, And A. Rosen (2013): "Intersection Bounds: Estimation and Inference," arXiv:0907.3503v3 [math.ST], forthcoming Econometrica.

Chesher, A. (2003): "Identification in Nonseparable Models," Econometrica, 71(5), 1405-1441.

— (2005): "Nonparametric identification under discrete variation," Econometrica, 73(5), 15251550.

Chesher, A., A. Rosen, And K. Smolinski (2011): "An Instrumental Variable Model of Multiple Discrete Choice," CeMMAP Working Paper CWP39/11.

Chesher, A., And K. Smolinski (2010): "Sharp Identified Sets for Discrete Variable IV Models," CeMMAP Working Paper CWP11/10.

D'Haultfoeuille, X. (2011): "On the Completeness Condition in Nonparametric Instrumental regression," Econometric Theory, 27, 460-471.

Doksum, K. (1974): "Empirical probability plots and statistical inference for nonlinear models in the two-sample case," Ann. Statist., 2, 267-277.

Durrett, R. (1996): Probability: Theory and Examples. Duxbury Press, Belmont, CA, second edn. 
Ekeland, I., A. Galichon, And M. Henry (2010): "Optimal transportation and the falsifiability of incompletely specified economic models," Economic Theory, 42(2), 355-374.

Eren, O. (2009): "Does membership pay off for covered workers? A distributional analysis of the free rider problem," Industrial and Labor Relations Review, 62(3), 367-380.

Forbes, S. J. (2008): "The effect of air traffic delays on airline prices," International Journal of Industrial Organization, 26(5), 1218-1232.

Froelich, M., AND B. Melly (2008): "Unconditional Quantile Treatment Effects under Endogeneity," IZA Discussion Paper No. 3288.

Gagliardini, P., ANd O. Scaillet (2012): "Nonparametric Instrumental Variable Estimation of Structural Quantile Effects," Econometrica, 80(4), 1533-1562.

Galichon, A., AND M. Henry (2009): "A test of non-identifying restrictions and confidence regions for partially identified parameters," Journal of Econometrics, 152(2), 186-196.

- (2011): "Set identification in models with multiple equilibria," Review of Economic Studies, $78(4), 1264-1298$.

Gandhi, A. (2008): "On the Nonparametric Foundations of Discrete Choice Demand Estimation," Discussion paper, University of Wisconsin-Madison.

Graddy, K. (1995): "Testing for Imperfect Competition at the Fulton Fish Market," Rand Journal of Economics, 26(1), 75-92.

Hausman, J. A. (1977): "Errors in variables in simultaneous equation models," J. Econometrics, 5(3), 389-401.

Hausman, J. A., And J. G. Sidak (2004): "Why Do the Poor and the Less-Educated Pay Higher Prices for Long-Distance Calls?," Contributions to Economic Analysis \&3 Policy, Vol. 3, No. 1, Article 3. http://www.bepress.com/bejeap/contributions/vol3/iss1/art3.

Heckman, J., And R. RobB (1986): "Alternative Methods for Solving the Problem of Selection Bias in Evaluating the Impact of Treatments on Outcomes," in Drawing Inference from Self-Selected Samples, ed. by H. Wainer, pp. 63-107. Springer-Verlag, New York.

Heckman, J. J., AND J. Smith (1997): "Making the most out of programme evaluations and social experiments: accounting for heterogeneity in programme impacts," Rev. Econom. Stud., 64(4), 487-535.

Hong, H., AND E. Tamer (2003): "Inference in Censored Models with Endogenous Regressors," Econometrica, 71(3), 905-932.

Honore, B., And L. Hu (2004): "On the Performance of Some Robust Instrumental Variables Estimators," Journal of Business and Economic Statistics, 22(1), 30-39.

Hoogerheide, L. F., J. F. KaAshoek, And H. K. van DiJk (2007): "On the shape of posterior densities and credible sets in instrumental variable regression models with reduced rank: An application of flexible sampling methods using neural networks," Journal of Econometrics, 139(1), 154-180.

Horowitz, J. L., AND S. LEE (2007): "Nonparametric instrumental variables estimation of a quantile regression model," Econometrica, 75(4), 1191-1208.

Imbens, G. W., And J. D. Angrist (1994): "Identification and Estimation of Local Average Treatment Effects," Econometrica, 62(2), 467-475.

Imbens, G. W., And W. K. Newey (2009): "Identification and Estimation of Triangular Simultaneous Equations Models Without Additivity," Econometrica, 77(5), 1481-1512. 
Jun, S. J. (2008): "Weak Identification Robust Tests in an Instrumental Quantile Model," Journal of Econometrics, 144, 118-138.

Koenker, R. (2005): Quantile Regression. Cambridge University Press.

Koenker, R., And G. S. Bassett (1978): "Regression Quantiles," Econometrica, 46, 33-50.

Koenker, R., And O. Geling (2001): "Reappraising Medfly longevity: A Quantile Regression Survival Analysis," J. Amer. Statist. Assoc., 96, 458-468.

Koenker, R., AND L. MA (2006): "Quantile regression methods for recursive structural equation models," Journal of Econometrics, 134(2), 471-506.

Kostov, P. (2009): "A spatial quantile regression hedonic model of agricultural land prices," Spatial Economic Analysis, 4(1), 53-72.

LAmarche, C. (2011): "Measuring the incentives to learn in Columbia using new quantile regression approaches," Journal of Development Economics, 96, 278-288.

LEE, S. (2007): "Endogeneity in quantile regression models: A control function approach," Journal of Econometrics, 141(2), 1131-1158.

Lehmann, E. L. (1974): Nonparametrics: statistical methods based on ranks. Holden-Day Inc., San Francisco, Calif.

MARmer, V., AND S. SAKATA (2012): "Instrumental variables estimation with weak-identificationrobust inference based on conditional quantile restriction," Working Paper.

Mas-Colell, A. (1979): "Homeomorphisms of compact, convex sets and the Jacobian matrix," SIAM J. Math. Anal., 10(6), 1105-1109.

Maynard, A., AND J. QIU (2009): "Public insurance and private savings: Who is affected and by how much?," Journal of Applied Econometrics, 24(2), 282-308.

Newey, W. K., AND J. L. Powell (2003): "Instrumental Variable Estimation of Nonparametric Models," Econometrica, 71, 1565-1578.

Rothenberg, T. J. (1971): "Identification in parametric models," Econometrica, 39, 577-591.

SAKATA, S. (2007): "Instrumental Variable Estimator Based on Conditional Median Restriction," Journal of Econometrics, 141(2), 350-382.

SAntos, A. (2012): "Inference in Nonparametric Instrumental Variables With Partial Identification," Econometrica, 80(1), 213-275.

Somainiy, P. (2012): "Competition and Interdependent Costs in Highway Procurement," Working Paper, Stanford.

Stock, J. H., J. H. Wright, AND M. Yogo (2002): "A Survey of Weak Instruments and Weak Identification in Generalized Method of Moments," Journal of Business and Economic Statistics, 20(4), $518-529$.

Torgovitsky, A. (2012): "Identification of Nonseparable Models with General Instruments," Working Paper.

van der VaArt, A. W., AND J. A. Wellner (1996): Weak Convergence and Empirical Processes. Springer Series in Statistics.

Wehby, G. L., J. C. Murran, E. E. Castilla, J. S. Lopez-Camelo, And R. L. Ohsfeldt (2009): "Quantile effects of prenatal care utilization on birth weight in Argentina," Health Economics, 18(11), $1307-1321$. 